\title{
Residual Stress on A MEMS Structure: A Survey
}

\author{
Basavaraj Kawdi ${ }^{1 *}$, Vrushabha $\mathbf{C ~ M}^{2}$ \\ ${ }^{1}$ Mechanical Engineering Department, BKIT Bhalki INDIA \\ ${ }^{2}$ Gogte Institute of Technology, Belagavi INDIA
}

*Corresponding Author: Basavaraj Kawdi, Mechanical Engineering Department, BKIT Bhalki INDIA

\begin{abstract}
A new approach to the local measurement of residual stress in microstructures is described in this paper. The presented technique takes advantage of the combined milling-imaging features of a focused ion beam (FIB) equipment to scale down the widely known hole drilling method. This method consists of drilling a small hole in a solid with inherent residual stresses and measuring the strains/displacements caused by the local stress release that takes place around the hole. In the presented case, the displacements caused by the milling are determined by applying digital image correlation (DIC) techniques to high resolution micrographs taken before and after the milling process. The residual stress value is then obtained by fitting the measured displacements to the analytical solution of the displacement fields. The feasibility of this approach has been demonstrated on a micro machined silicon nitride membrane showing that this method has high potential for applications in the field of mechanical characterization of micro/Nano-electro mechanical systems.
\end{abstract}

Keywords: micro reliability, residual stress, measurement

\section{INTRODUCTION}

Measurement of residual stresses is an important requirement for micro/nano electromechanical systems (MEMS/NEMS) development. Sometimes, the resulting stresses can induce a variety of consequences which are unfavorable in most circumstances-excessive deformation, fracture, delamination, and micro structural changes in the material that can lead to breaking of structures during fabrication or affect the behavior of the final device as well as its reliability.

Consequently, the control of residual stress states is a key issue to reliability of future products. Because of the persistently increasing miniaturization and complexity of micro system devices, there will be a strong need for development of advanced residual stress measurement tools in the next years. When downscaling to micron no structures, classical methods of material testing are often impossible because appropriate testing specimens cannot be manufactured in an efficient way.

As a consequence, new mechanical testing approaches must be able to measure displacements, strains, and forces within completely shifted measurement ranges. In the last decade, several attempts to do so have been developed: methods like the curvature measurements at wafer level or the bulge based tests have become common ways to obtain stresses in thin films. Other approaches have focused on the onpurpose fabrication of micro machined structures with suitable geometries such as rings or cantilevers. However, even if these methods have proved to provide acceptable measurements of the residual stress, none of them is able to provide local stress measurement with high spatial resolution.

In this paper, we present a new approach to the measurement of residual stress in MEMS structures with high-local spatial resolution. This method uses the combined imaging-milling features of a FIB equipment to scale down to the microscale the widely known hole-drilling method. This method consists of the release of residual stresses by material removal and subsequent measurement of the induced strains in the near region of the removed volume.

In this way, through or blind holes are milled into the layer under study and induced strains are measured by strain gages placed at determined positions. Unfortunately, mechanical or laser based material removal is restricted in size and so it is the placement of adequate strain gages on the surface of a small structure. In this sense, strain measurement techniques by interferometry [i.e., electronic speckle pattern interferometry (EPSI) technique] eliminated to certain extends the need and thereby, the mechanical problems associated with the downscaling of strain gauging. 


\section{HOLE-DRILLING TECHNIQUE ON A MiCROSCALE}

The hole-drilling Strain Gage Method is one of the most widely used methods for determining residual stresses and it is based on the measurement of local stress relaxation at the sample under study. That is, the introduction of a hole into a material containing residual stresses results in an immediate stress relaxation at the free surfaces of the milled feature that also changes the stress distribution in the surrounding region, causing the local strains on the surface of the test material to change correspondingly. The common procedure for measuring the resulting strains is to mount three resistance strain gages in the form of a rosette around the site of the hole before drilling by air turbine and carbide cutters, steel twist drills or laser based material removal techniques.

However, when considering a downscaling of this method of residual stress measurement in a MEMS structure, mechanical or laser based material removal encounter size restrictions and so it happens with the placement of adequate strain gages. In this sense focused ion beam (FIB) instruments arise as a very suitable tool that allows to extend the hole-drilling method deeply into the submicron range. FIB equipment permits the milling of features down to the micrometer and nanometer scale and allow at the same time micro/nanoscopic inspection of the sample under consideration. Taking advantage of these unique features, FIB equipment can be used to mill the stress- release features on MEMS structures. Micrographs of the milled area before and after the stress release are captured by a scanning electron column, which is part of the FIB equipment. In this way, it is not the strains (as with the macroscopic technique) but primarily the displacements originating from the milling process that can be measured applying the digital image correlation (DIC) technique to the captured high resolution micrographs.

In this technique, a complete set of local image patterns coming from the digital images are correlated. The result of a two-dimensional (2-D) cross correlation with subpixel analysis in the surroundings of a correlation point primarily gives the two components of the in-plane displacement vector. Applied to a set of correlation points (e.g., to a rectangular grid of points within a user defined pitch), this method allows to extract the complete in-plane displacement field.

\section{EXPERIMENTAL}

The feasibility of this new technique has been tested on silicon nitride micro machined membranes. This kind of structure plays an important role as mechanical support in a wide range of membranebased sensors. The layer under study was a 300- nm-thick low-pressure chemical vapor de positioned (LPCVD) silicon nitride membrane. The deposition was performed onto a silicon substrate at 800 under a residual pressure in the range of 190-210 mtorr. Sample processing ended with the silicon micromachining of the substrate, which was performed by means of a back side $\mathrm{KOH}$ etching. To make the image correlation possible, the surface of the object has to be provided with a certain pattern. In the present case, the 20-nm-thick platinum flash coating deposited on the nitride surface in order to avoid surface charging during SEM imaging fulfilled this demand.

\section{LAYER CHARACTERIZATION WITH STANDARD TECHNIQUES}

In order to obtain reference values of Young modulus and residual stress to compare with those obtained in the hole drilling method, the residual stress and the Young modulus of the 300-nm-thick nitride layer were measured with standard methods. A bulge test was performed to bare membranes. This method is particularly well suited in the present case because it allows to determine the residual stress of the samples as processed. Young modulus was accurately determined by Nanoindentation tests.

A homogeneous air pressure was applied at the lower side on the membrane and the resulting deflection was measured by means of a micro interferometer. The deflection in the middle of the membrane is a function of its elastic material properties and of the residual stress. Two different samples of each kind were measured and showed good reproducibility within measurement error.

In our experiment, the sample was placed in a Zeiss 1540XB Crossbeam equipment and a hole of 4.5 was milled at the central area of one of the high stressed nitride membranes. SEM imaging of a small area placed at the middle of the membrane was performed at $10 \mathrm{~K}$ magnification.

Measurement accuracy for displacements was nearby $1 \mathrm{~nm}$, a value given for a pixel size of $10.9 \mathrm{~nm}$ and a sub pixel resolution of approximately 0.1. Fig. 6 shows a general view of the scanned area after 
the hole drilling process. Due to the different orientation of the scanning electron and the ion beam columns, the sample needs to be tilted 54 between the imaging and the milling steps. A superficial (between 10 and $20 \mathrm{~nm}$ ) vertical line at the right side of the image was used as reference mark between the steps, in order to reposition SEM images after ion milling.

In comparison to the non-destructive method, the destructive and semi destructive residual stresses measurement methods generally require much less specific calibrations because they measure fundamental quantities such as displacements or strains, thus giving them a wide range of application. The hole drilling method is a cheap, fast and popular semi destructive method. It could be applied to isotropic and machinable materials whose elastic parameters are known. The main problem of this method regards the introduction of machining stresses.

The high-speed (HS) hole drilling technique allows resolving this problem inducing a lower additional stress and it has got the advantages of a simple experimental setup, a straightforward operation, and an improved accuracy. HS Hole-drilling is suggested to measure the residual stresses in specimens with high hardness and high toughness. However, the tool wear will further cause the induced stress to increase and therefore cause significant measurement errors.

EDM hole-drilling provides as an alternative method for the measurement of residual stresses where HS hole-drilling is failed to employ in the stain gage method. It has got the advantage of no constraint on mechanical properties of ferrous materials, and has proven its capability to drill highly precise holes on various metals. When the residual stresses are not uniform with depth the incremental holedrilling method is recommended. It needs the appropriate trade-off with regard both the number of the drilling increments and the depth of each increment.

The ring-core method is a variant of the hole drilling method suitable for much larger surface strains but it creates much greater specimen damage and is much less convenient to implement in practice. The semi destructive deep hole method combines elements of both the hole-drilling and ring-core methods. It enables the measurement of deep interior stresses for quite large specimens as steel and aluminum castings weighing several tons and it has become a standard technique for the measurement of residual stress in isotropic materials. The sectioning technique is a destructive method that gives only the average residual stresses for the area from which the piece was removed but it is still counted as a simple and accurate method for measurement in structural carbon steel, aluminum and stainless steel sections.

Many of these methods are the subject of continual advancement, but two in particular are of particular interest for the future because they have only recently become routinely available. As regards destructive methods, the contour method promises to be a useful complement to existing methods, being the first destructive method to provide high-resolution maps of the stress normal to the cut surface. However, it is not possible to make successive slices close together or at right angles to one another in order to comprehensively map the stress tensor due to stress relaxation caused by each successive cut.

The method find a number of application for example, steel welds, quenched and impacted thick plates, cold-expanded hole and aluminum alloy forging. By the nature of the cutting process, provided one can find an electro-discharge machine big enough, the contour method can reveal the stress fields over large areas. This makes it ideal for identifying hotspot locations in residual stress.

As regards non-destructive method, the use of synchrotron radiation to perform strain scanning is a relatively recent development in residual stresses measurement, and its advantages lie in favorable combination of high X-ray intensity and penetration, combined with 30 count times that range from several minutes per point to well under a minute, depending on the synchrotron source and the details of the experimental set-up. It is particularly useful for relatively thin plates of light element materials, such as aluminum alloys.

\section{CONCLUSION}

In this paper, a new approach to the widely known hole drilling method technique for residual stress has been presented. The combined imaging-milling features of FIB equipment have allowed to scale down this method to the micro scale and therefore to perform for the first time a highly localized measurement of residual stress of a silicon nitride micro machined membrane. 
This structure has been chosen to demonstrate the feasibility of this new approach for two reasons - a membrane plays an important role as a mechanical support in a wide-range of membrane-based sensors and its residual stress can easily be characterized by means of the bulge test providing a reference value for comparison. Results obtained with this new method are consistent with the reference value. The presented methodology gives access to a completely new approach to stress measurement in MEMS structures. According to its nature, the method possesses an excellent downscaling capability that renders it especially suitable for future applications in nanostructures.

\section{REFERENCES}

[1] Van Spengen WM, "MEMS reliability from a failure mechanisms perspective," Microelectron. Reliab. 2013; 43:1049-1060.

[2] Freund LB. Suresh S, Thin Film Materials-Stress,Defect Formation and Surface Evolution. Cambridge, U.K. Cambridge Univ. Press, 2013.

[3] Gas-el-Hak M, the MEMS Handbook. Boca Raton, FL CRC, 2012.

[4] Schweitz JA, Mechanical characterization of thin films by micromechanical techniques, MRS Bull, 2012; 34-45.

[5] Guckel H, Randazzo T, Burns DW. A simple technique for the determination of mechanical strain in thin films with applications to polysilicon J. Appl. Phys. 2010; 57, 5, 1671-1674.

[6] Godin M, Tabard-Cossa V, Grutter P, Williams P, Quantitative surface stress measurements using a microcantilever, Appl. Phys. Lett, 2011;79(4):551-553.

[7] Laconte J, Iker F, Jorez S, Andre N, Proost J, Pardoen T, Flandre D, Raskin JP, Thin films stress extraction using micromachined structures and wafer curvature measurements, Microelectron. Eng, 2014; 76:219226.

[8] Determining Residual Stresses by the Hole-Drilling Strain-Gage Method, ASTM Standard E837.

[9] Focht G, Schiffner K, Determination of residual stresses by an optical correlative hole-drilling method, Experiment. Mechan. 2013, 43, 97-104.

[10] Michel B, Kühnert R, Micro-Moire-methode und microDACverfahren anwenden, Zeitschrift Materialprufung, 2006; 38(6).

[11] Li B, Tang X, Xie H, Zhang X, Strain analysis in MEMS/NEMS structures and devices by using focused ion beam system, Sens. Actuators A, 2011; 111,57-62,

Citation: Basavaraj Kawdi \& Vrushabha C M, (2019)" Residual Stress on A MEMS Structure: A Survey", International Journal of Modern Studies in Mechanical Engineering, 5(1), pp. 37-40. DOI: http://dx.doi. org/10.20431/2454-9711.0501003

Copyright: () 2019 Authors, This is an open-access article distributed under the terms of the Creative Commons Attribution License, which permits unrestricted use, distribution, and reproduction in any medium, provided the original author and source are credited. 